\title{
Ações de educação em saúde como estratégia de prevenção e de controle das
}

\section{parasitoses intestinais: um estudo de revisão sistemática da literatura}

\author{
Health education actions as a prevention and control strategy for intestinal parasites: a systematic
} literature review study

Acciones de educación en salud como estrategia de prevención y control de la parasitosis intestinal: un estudio de revisión sistemática de la literatura

Recebido: 10/08/2021 | Revisado: 18/08/2021 | Aceito: 23/08/2021 | Publicado: 25/08/2021

\author{
Welida Carvalho Vasconcelos \\ ORCID: https://orcid.org/0000-0002-2325-2089 \\ Universidade Federal do Rio De Janeiro, Brasil \\ E-mail: welidapesquisa@gmail.com \\ Adenildo da Silva-Vasconcelos \\ ORCID: https://orcid.org/0000-0002-2138-6542 \\ Universidade Federal do Estado do Rio de Janeiro, Brasil \\ E-mail: asvpesquisa@gmail.com
}

\begin{abstract}
Resumo
No Brasil, parasitoses intestinais representam importante problema de saúde pública, sendo endêmicas em diversas regiões, especialmente naquelas onde as condições de vida estão influenciadas negativamente por fatores decorrentes da pobreza, das precárias condições de habitação, do deficiente abastecimento de água potável, saneamento básico e déficit educacional populacional. O Ministério da Saúde estabeleceu o desenvolvimento de ações de Promoção da Saúde, por meio da educação em saúde, visando criar estratégias de prevenção e controle para reduzir a prevalência, morbidade e mortalidade por enteroparasitoses no país. Este estudo objetivou identificar, na literatura científica brasileira, as ações de Promoção da Saúde realizadas para prevenção e controle de parasitoses intestinais no país. Trata-se de revisão sistemática da literatura de 18 artigos levantados nas plataformas MEDLINE, SciELO, Biblioteca Virtual em Saúde, Portal de Periódicos Capes e base de dados online Google Scholar publicados no período de 2015 a 2020. A análise das publicações permitiu inferir que ações de educação em saúde são um instrumento profilático efetivo para o aprendizado sobre as parasitoses intestinais, seus agentes causadores e sobre os modos de prevenção e de controle. Essas ações são de baixo custo e facilmente desenvolvidas por profissionais de qualquer formação, em diferentes contextos, realidades populacionais e espaços de atuação profissional, como escolas, Unidade Básica de Saúde, principalmente com populações infanto-juvenis por meio da utilização de atividades educativas lúdicas, jogos, teatro, música, literatura, montagem macromodelos e demonstrações de espécimes preservados.
\end{abstract}

Palavras-chave: Parasitoses intestinais; Enteroparasitoses; Promoção da saúde; Educação em saúde.

\begin{abstract}
In Brazil, intestinal parasites represent an important public health problem, being endemic in several regions, especially those where living conditions are negatively influenced by factors resulting from poverty, precarious housing conditions, poor drinking water supply, basic sanitation and population educational deficit. The Ministry of Health established the development of Health Promotion actions, through health education, aiming to create prevention and control strategies to reduce the prevalence, morbidity and mortality of intestinal parasites in the country. This study aimed to identify, in the Brazilian scientific literature, the Health Promotion actions carried out for the prevention and control of intestinal parasites in the country. This is a systematic literature review of 18 articles collected on the MEDLINE, SciELO, Virtual Health Library, Capes Journal Portal and Google Scholar online database platforms published from 2015 to 2020 . The analysis of the publications allowed us to infer which actions Health education programs are an effective prophylactic tool for learning about intestinal parasites, their causative agents and about ways of prevention and control. These actions are low-cost and easily developed by professionals of any training, in different contexts, population realities and spaces of professional activity, such as schools, Basic Health Unit, especially with children and young people through the use of playful educational activities, games, theater, music, literature, macromodel assembly and preserved specimen demonstrations.
\end{abstract}

Keywords: Intestinal parasitosis; Enteroparasitosis; Health promotion; Health education. 


\begin{abstract}
Resumen
En Brasil, los parásitos intestinales representan un importante problema de salud pública, siendo endémicos en varias regiones, especialmente aquellas donde las condiciones de vida están influenciadas negativamente por factores derivados de la pobreza, las precarias condiciones de vivienda, el mal abastecimiento de agua potable, el saneamiento básico y el déficit educativo de la población. El Ministerio de Salud estableció el desarrollo de acciones de Promoción de la Salud, a través de la educación en salud, con el objetivo de crear estrategias de prevención y control para reducir la prevalencia, morbilidad y mortalidad de los parásitos intestinales en el país. Este estudio tuvo como objetivo identificar, en la literatura científica brasileña, las acciones de Promoción de la Salud realizadas para la prevención y control de parásitos intestinales en el país. Se trata de una revisión bibliográfica sistemática de 18 artículos recopilados en las plataformas de bases de datos en línea MEDLINE, SciELO, Virtual Health Library, Capes Journal Portal y Google Scholar publicados entre 2015 y 2020. El análisis de las publicaciones permitió inferir que las acciones de educación para la salud son una herramienta profiláctica eficaz para el conocimiento de los parásitos intestinales, sus agentes causantes y las formas de prevención y control. Estas acciones son de bajo costo y fácilmente desarrolladas por profesionales de cualquier formación, en diferentes contextos, realidades poblacionales y espacios de actividad profesional, como escuelas, Unidad Básica de Salud, especialmente con niños y jóvenes mediante el uso de actividades educativas lúdicas, juegos, teatro, música, literatura, montaje de macromodelos y demostraciones de ejemplares conservados.
\end{abstract}

Palabras clave: Parasitosis intestinal; Enteroparasitosis; Promoción de la salud; Educación para la salud.

\title{
1. Introdução
}

As parasitoses representam importante problema de saúde pública mundial, ocorrendo principalmente nos países em desenvolvimento. No Brasil essas infecções se comportam de forma endêmica em diversas regiões, contribuindo para ampliação de sérios problemas sociais e econômicos (Melo et al., 2004; Busato et al., 2014).

As infecções por parasitas intestinais podem ser causadas por protozoários, sendo os mais frequentes Entamoeba histolytica Schaudinn, 1903 (Protozoa, Endamoebidae), Endolimax nana Wenyon e O 'Connor, 1917 (Protozoa, Endamoebidae) e Giardia intestinalis Lambl, 1859 (Sin. Giardia lamblia e Giardia duodenalis; Protozoa, Hexamitidae) ou por helmintos, principalmente os Nematoda Ascaris lumbricoides Linnaeus, 1758 (Nemathelminthes, Ascarididae), Enterobius vermicularis Linnaeus, 1758 (Nemathelminthes, Oxyuridae), Trichuris trichiura Roederer, 1761 (Nemathelminthes, Trichuridae), Strongyloides stercoralis Bavay, 1876 (Nemathelminthes, Strongyloididae), Necator americanus Stiles, 1902 (Nemathelminthes, Ancylostomatidae) e Ancylostoma duodenale Dubini, 1843 (Nemathelminthes, Ancylostomatidae); e os Cestoda Taenia solium Linnaeus, 1758 (Plathyhelminthes, Taeniidae), Taenia saginata Linnaeus, 1758 (Plathyhelminthes, Taeniidae), Hymenolepis nana Stiles, 1906 (Plathyhelminthes, Hymenolepididae) e Hymenolepis diminuta Rudolphi, 1819 (Plathyhelminthes, Hymenolepididae) (Rey, 2008; Andrade et al., 2010; UFRRJ, Neves, 2016).

O Brasil apresenta inúmeras condições propícias para a ocorrência e manutenção de diferenciadas parasitoses, sendo as mais comuns a amebíase, a giardíase, a ascaridíase, a ancilostomíase, a enterobiose, a teníase, a esquistossomose, a malária e a Doença de Chagas (Melo et al., 2004; Rey, 2008; Busato et al., 2014).

A literatura tem destacado a importância de diversos fatores econômicos, políticos, sociais, ambientais e climáticos para a ocorrência das parasitoses. Fatores como crescimento desordenado das cidades, condições de vida da população (habitações precárias, abastecimento de água potável ineficiente ou inexistente, pobreza) e o déficit educacional populacional, são relacionados como os principais responsáveis pela manutenção das parasitoses. Essas condições, de modo geral, favorecem a disseminação de ovos e larvas infectantes de helmintos e cistos de protozoários, que são transmitidos por via orofecal, pela ingestão de água e alimentos contaminados, via cutânea, mãos e/ou poeira contaminada (Melo et al., 2004, Basso et al., 2008; Souza et al., 2015; Busato et al., 2014; Moraes et al., 2019). Favorecem também a proliferação de vetores (Rey, 2008).

De um modo geral, as parasitoses são de fácil tratamento, principalmente na Atenção Básica à Saúde, e preveníveis, especialmente através de ações de Promoção da Saúde - PS (Andrade et al., 2010, Silva et al., 2014). Entretanto, essas infecções ainda fazem parte do grupo de doenças referidas como negligenciadas, o que justifica a persistência delas na população, principalmente devido a insuficiente oferta de investimentos em políticas públicas que garantam a melhoria da 
qualidade de vida da população, tais como o saneamento básico de qualidade nas cidades (Ludwig et al., 1999; Mackey et al., 2014).

Essa realidade, aliada às precárias condições de higiene nas comunidades e ausência de informações significativas e contextualizadas sobre as doenças, os parasitos e sobre as medidas preventivas são fatores que também contribuem para a ocorrência das parasitoses intestinais junto às comunidades, sobretudo em crianças em idade escolar e populações menos favorecidas (Munareto et al., 2021). Por essas questões as parasitoses intestinais são consideradas doenças relacionadas a pobreza (Basso et al., 2008; Silva et al., 2014), mesmo que venham acontecendo melhorias na oferta de água potável, instalações de saneamento básico e campanhas de tratamento paliativo em massa conduzidas nos diversos locais do Brasil. O fato é que as precárias condições de vida contribuem para uma alta suscetibilidade as parasitoses (Mackey et al., 2014).

No Brasil, as parasitoses intestinais estão inseridas na agenda de discussão sobre os modelos de prevenção e controle de doenças, visto que sua ocorrência pode desencadear problemas de saúde graves como a desnutrição, a anemia, problemas de desenvolvimento, dentre outros (Melo et al., 2004; Moraes et al., 2019). Nessa perspectiva, no ano de 2005, o Ministério da Saúde estabeleceu o Plano Nacional de Vigilância e Controle das Enteroparasitoses com o objetivo de definir as estratégias de controle dos fatores de risco associados, de modo a reduzir a prevalência, a morbidade e a mortalidade por enteroparasitoses no país. Desde então, o meio pelo qual essas estratégias são colocadas em prática ocorre, principalmente, através da produção e divulgação de conhecimento sobre o comportamento epidemiológico das doenças, os agentes etiológicos prevalentes e principais fatores de risco, do hospedeiro e do meio ambiente, para ocorrência das infecções, e, também através do desenvolvimento de ações de Promoção da Saúde para a população, por meio da educação em saúde (Brasil, 2005).

A educação em saúde é o processo de ensino-aprendizagem que visa a Promoção da Saúde, possibilitando o desenvolvimento da conscientização individual e coletiva relacionada às responsabilidades e ao direito à saúde. As práticas educativas em saúde são importantes, pois, quando bem construídas, garantem o acesso às informações necessárias para a valorização e incorporação de hábitos saudáveis, a construção de conhecimento preventivo e valorização do indivíduo e coletividades, além das suas habilidades para a construção de respostas aos problemas pessoais e da comunidade. No entanto, em relação às doenças parasitárias, as ações de educação em saúde acontecem de modo generalizado e descontextualizado dos conteúdos do cotidiano das pessoas, não produzindo efeito reflexivo sobre como os modos de vida auxiliam na perpetuação das doenças no ambiente (Silva et al., 2014).

A literatura mostra que, apesar do tema das doenças parasitárias fazer parte do currículo escolar de ciências, há uma tendência em haver pouca discussão ou um desmerecimento sobre o tema no âmbito escolar. Os estudos indicam que há pouca ou nenhuma referência aos ciclos de vida dos parasitos, o que acaba por impedir o acesso a informações corretas sobre as formas infectantes e os locais no ambiente onde as mesmas podem habitar. Ademais, todo esse quadro dificulta a Promoção da Saúde para o entendimento das parasitoses como um problema de saúde pública relacionado às condições de vida precárias e também reduz a construção do conhecimento correto sobre os parasitos (Toscani et al., 2007; Silva et al., 2014; Trindade et al., 2015).

As relações entre saúde e condições de vida da população e os meios pelos quais possamos redirecionar as práticas de saúde, tem recebido suporte nos pressupostos da Promoção da Saúde. Assim, de modo a vencer e contrapor modelos assistenciais em Saúde Pública, a Promoção da Saúde permite a ideia de indivíduos autônomos, capacitados e atuantes na construção de respostas sociais sobre sua vida e sua saúde, sem, no entanto, tirar a responsabilidade do estado em garantir as melhorias necessárias para a qualidade de vida da população (Czeresnia \& Freitas, 2003; Brasil, 2010).

A Promoção de Saúde ganha destaque no campo da Saúde Pública a partir da década de 1980, quando a Organização Mundial da Saúde (OMS) inaugura seu conceito: "processo de capacitação da comunidade para atuar na melhoria da sua qualidade de vida e saúde, incluindo maior participação no controle desse processo" (Brasil, 2010). A Carta de Ottawa, marco 
importante à efetivação desse conceito, traz a necessidade de uma atuação responsável de todos os setores sociais, em seus diferentes níveis, pela saúde e pela compreensão das consequências de ações equivocadas sobre a saúde da população (Brasil, 2002; Sícoli \& Nascimento, 2003).

Dentre as ações tratadas pela carta de Otawa estão: a criação de ambientes favoráveis à saúde, ou seja, que se reconheçam os impactos que as mudanças no meio ambiente têm sobre a saúde e a importância da proteção ambiental; o reforço da ação comunitária, que possam garantir a participação popular nas discussões dos assuntos de saúde e acesso contínuo à informação; e a disponibilização de informações corretas e a educação para a saúde em todas as fazes da vida, nos diferentes espaços coletivos. Esse conjunto de ações faz parte das estratégias de promoção preconizadas pela sua Política Nacional de Promoção da Saúde (Brasil, 2002; 2010).

Buss (2009) corrobora com esse entendimento ao descrever que a concepção de Promoção da Saúde, como vem sendo construída ao longo dos anos, parece ser um caminho ótimo para o enfrentamento dos diversos problemas que permeiam a vida das populações. Dessa forma, com suporte na concepção ampliada sobre o processo saúde-doença e de seus determinantes, a Promoção da Saúde indica a possibilidade de articulação entre os conhecimentos científicos e populares, além de permitir a mobilização de diferentes recursos intersetoriais como forma articulada para enfrentamento e resolução dos problemas, especialmente aqueles tidos como negligenciados, como as parasitoses intestinais.

As estratégias de Promoção da Saúde como resposta a uma demanda de indivíduo e/ou a uma instituição ou em resposta a uma demanda própria, em que o sujeito mobilizou-se para atuar diretamente nos problemas, caminham junto com a análise ampliada dos problemas sociais que interferem na saúde. Dessa forma, essas estratégias caminham junto também com a adoção de práticas cuidadoras e integrais que sejam atentas ao foco principal que é a melhoria na qualidade de vida e a redução dos riscos. Elas, então atuam de modo a reorientar o foco no individual, incentivando a construção de políticas públicas que sejam efetivas não só no nível unitário, mas também no nível coletivo (Czeresnia \& Freitas, 2003).

Isto posto, é oportuno realçar a importância que informações sobre a produção científica existente trazem para se estimar a preocupação e a atuação de profissionais pesquisadores e órgãos interessados na melhoria das ações de Promoção da Saúde em suas diferentes dimensões e contextos de atuação. Dessa forma, considerando a relação intrínseca entre Promoção da Saúde e controle de doenças parasitárias, indagou-se: Como se caracterizam as ações de Promoção da Saúde realizadas para a prevenção e o controle de parasitoses intestinais no Brasil?

O presente estudo teve como objetivo identificar, na literatura científica brasileira, as ações de Promoção de Saúde realizadas para prevenção e controle de parasitoses intestinais no Brasil.

\section{Metodologia}

Estudo bibliográfico descritivo do tipo revisão sistemática da literatura, a qual se constitui método específico, focada em questão bem definida, que tem como objetivo a identificação, seleção, avaliação e síntese das evidências relevantes disponíveis (Galvão; Pereira, 2014). O levantamento das publicações ocorreu em três momentos distintos: agosto a outubro de 2017; agosto a outubro de 2019, e agosto a outubro de 2020, de modo a possibilitar a inclusão de uma amostra variada e qualitativamente significativa sobre a temática analisada.

A busca da literatura foi realizada nos bancos de dados informatizados da Biblioteca Virtual em Saúde-BVS, nas bases de dados da Literatura Internacional em Ciências da Saúde-MEDLINE via PubMed, do Scientific Eletronic Library Online (SciELO), no Portal de Periódicos Capes e, de modo complementar, a base de dados online Google Scholar, considerando que grande parte das produções científicas também podem ser encontradas através da literatura cinzenta. Para tanto, foram aplicados os seguintes descritores e combinações, com o uso do operador booleano "and": "parasitoses intestinais" "promoção da saúde"; "parasitoses intestinais" "educação em saúde"; "enteroparasitoses" "promoção da saúde"; 
"enteroparasitoses" "atividades educativas", aplicados de acordo com as particularidades de cada base de dados, ou seja, nas suas versões em português para as bases BVS, Portal Capes, Scielo e Google Scholar, e em inglês para a base MEDLINE. Os descritores foram selecionados a partir da consulta no Descritores em Ciências da Saúde (DeCS) e os termos associados foram consultados no Medical Subject Headings (Mesh).

O período de busca considerou as publicações realizadas no Brasil nos anos de 2015 a 2020. A escolha do ano de 2015 se deve ao marco da reedição da Política Nacional de Promoção da Saúde. Foram selecionadas as publicações completas que fizessem referência, no título, resumo ou palavras-chave, ao objeto de estudo utilizando as palavras: parasitoses, enteroparasitoses, promoção da saúde, educação em saúde ou atividade educativa.

Para a seleção das publicações foram estabelecidos os seguintes critérios de inclusão: trabalhos relacionados à temática, disponíveis sob a forma de artigos, teses e dissertações, realizados no Brasil, nos anos referidos, descritos nas modalidades de relato de pesquisa, estudo teórico-prático e relato de caso de experiência profissional, em português, espanhol ou inglês, que descrevessem a experiência da prática de Promoção da Saúde. Com relação aos critérios de exclusão, foram desconsiderados os estudos não disponibilizados em seu formato completo.

Estas obras foram submetidas à fase de pré-seleção, em que tiveram seus resumos lidos de modo a permitir a apuração somente daquelas que versassem em sua maior parte sobre ações de Promoção da Saúde com foco nas enteroparasitoses. As obras que não atenderam esse critério foram desconsideradas para a próxima fase. A fase de seleção contou com leitura integral das publicações pré-selecionadas. Nesta fase, foram selecionadas aquelas publicações que versaram sobre tipos de ações de Promoção da Saúde para prevenção e controle das parasitoses. A seleção foi feita por um dos pesquisadores, sendo conferida posteriormente pelos dois pesquisadores responsaveis. Após essa seleção, aplicouse o diagrama do PRISMA, como exposto no fluxograma da Figura 1. 
Figura 1. Fluxograma da seleção dos estudos.

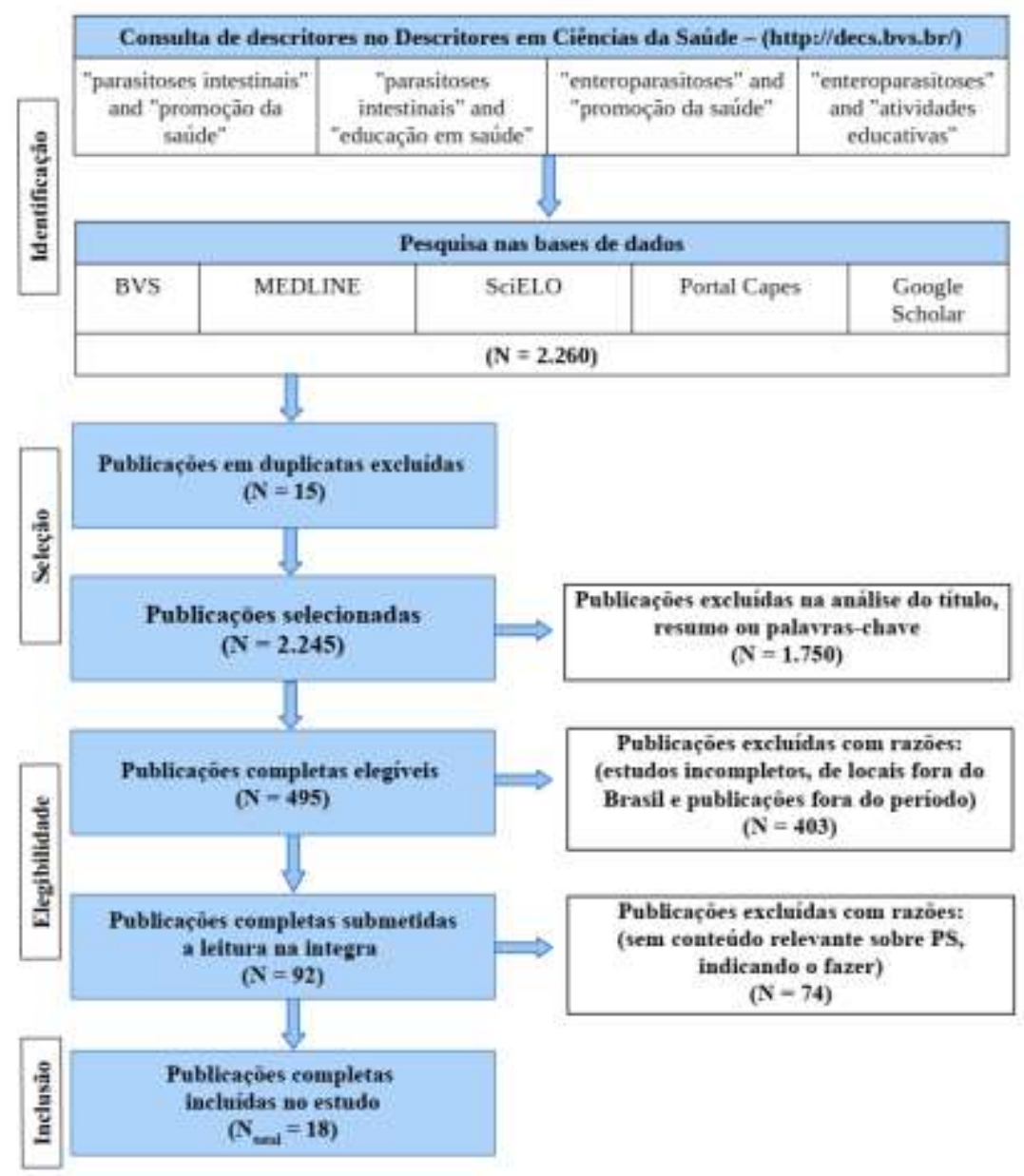

Fonte: Autores (2021).

\section{Resultados e Discussão}

Após a aplicação dos critérios de inclusão e exclusão, procedeu-se a análise das dezoito publicações na íntegra, com investigação de dados como ano e local de publicação, objetivo de estudo, espaço e público-alvo da ação, ações de promoção da saúde evidenciada. Os principais achados dos trabalhos identificados na presente busca sistemática da literatura são apresentados a seguir, em ordem cronológica de publicação (Quadro 1). 
Quadro 1. Distribuição das publicações sobre ações de Promoção de Saúde realizadas para prevenção e controle de parasitoses no Brasil, segundo ano e local de publicação, objetivo de estudo, espaço e público-alvo da ação, ações de promoção.

\begin{tabular}{|c|c|c|c|c|c|}
\hline $\begin{array}{l}\text { Ano/Local } \\
\text { de } \\
\text { publicação }\end{array}$ & Título & Autores & Objetivo do estudo & $\begin{array}{l}\text { Espaço/ } \\
\text { Público }\end{array}$ & Ações de Promoção da Saúde \\
\hline $\begin{array}{c}2015 \\
\text { Maranhão }\end{array}$ & $\begin{array}{c}\text { Prevenção a } \\
\text { parasitoses } \\
\text { ocasionadas por } \\
\text { cestodas através de } \\
\text { jogos lúdicos no } \\
\text { ensino } \\
\text { fundamental II }\end{array}$ & $\begin{array}{l}\text { Araújo, A.A. } \\
\text { et al. }\end{array}$ & $\begin{array}{l}\text { Desenvolver metodologias alternativas } \\
\text { para os conteúdos de Parasitologia } \\
\text { abordados na disciplina de Ciências do } \\
\text { Ensino Fundamental na Escola } \\
\text { Municipal Antenor Gomes Viana } \\
\text { Júnior, situada na cidade de Caxias, } \\
\text { MA, e a adequação desses conteúdos } \\
\text { às necessidades constatadas na } \\
\text { comunidade onde a escola está } \\
\text { inserida, visando aprimorar o processo } \\
\text { de ensino-aprendizagem de forma } \\
\text { dinâmica, lúdica e motivadora. }\end{array}$ & $\begin{array}{l}\text { Escola/ } \\
\text { Crianças }\end{array}$ & $\begin{array}{c}\text { Atividade Educativa de } \\
\text { construção de jogos lúdicos sobre } \\
\text { parasitoses para auxílio nas aulas } \\
\text { de ciências. }\end{array}$ \\
\hline $\begin{array}{c}2015 \\
\text { Mato Grosso }\end{array}$ & $\begin{array}{l}\text { Oficina educativa } \\
\text { sobre verminoses: } \\
\text { contribuição para } \\
\text { educação } \\
\text { profilática na } \\
\text { escola }\end{array}$ & $\begin{array}{l}\text { Henrique, } \\
\text { V.H.O. et al. }\end{array}$ & $\begin{array}{l}\text { Realizar oficinas, explanando sobre as } \\
\text { principais verminoses, sintomas e } \\
\text { profilaxias, promovendo assim um } \\
\text { trabalho de educação profilática. }\end{array}$ & $\begin{array}{l}\text { Escola/ } \\
\text { Crianças }\end{array}$ & $\begin{array}{c}\text { Oficinas expositivas com uso de } \\
\text { peças parasitológicas conservadas } \\
\text { em álcool } 70 \% \text {. }\end{array}$ \\
\hline $\begin{array}{l}2016 \\
\text { Rio de } \\
\text { Janeiro }\end{array}$ & $\begin{array}{l}\text { Conhecimentos } \\
\text { sobre parasitoses } \\
\text { intestinais como } \\
\text { estratégia para } \\
\text { subsidiar } \\
\text { ferramentas de } \\
\text { educação em saúde }\end{array}$ & Teixeira, F.A. & $\begin{array}{l}\text { Avaliar a frequência de parasitoses } \\
\text { intestinais e realizar práticas de } \\
\text { educação em saúde com pré-escolares } \\
\text { e profissionais da educação de duas } \\
\text { creches no estado do Rio de Janeiro, } \\
\text { Brasil. }\end{array}$ & $\begin{array}{l}\text { Escola/ } \\
\text { Adultos } \\
\text { e } \\
\text { Crianças }\end{array}$ & $\begin{array}{l}\text { Atividade Educativa em saúde } \\
\text { realizada através de exposição de } \\
\text { peças parasitológicas conservadas } \\
\text { em formol e grupo focal. }\end{array}$ \\
\hline $\begin{array}{l}\text { Rio de } \\
\text { Janeiro }\end{array}$ & $\begin{array}{c}\text { Parasitoses } \\
\text { intestinais em } \\
\text { escolares de } \\
\text { Niterói, RJ: } \\
\text { frequência, } \\
\text { conhecimentos e } \\
\text { educação em saúde }\end{array}$ & Siqueira, M.P. & $\begin{array}{l}\text { Avaliar a frequência de parasitoses } \\
\text { intestinais em estudantes e } \\
\text { funcionários de sete Escolas } \\
\text { Municipais de Niterói, RJ, Brasil, } \\
\text { identificar os saberes e implementar } \\
\text { ações educativas. }\end{array}$ & $\begin{array}{l}\text { Escola/ } \\
\text { Adultos } \\
\text { e } \\
\text { Crianças }\end{array}$ & $\begin{array}{c}\text { Atividade Educativa de } \\
\text { sensibilização da comunidade } \\
\text { escolar e feira educativa com } \\
\text { banners e dinâmicas de } \\
\text { observação de helmintos adultos } \\
\text { em frascos, construção de } \\
\text { modelos em massas de biscuit, } \\
\text { pesca e pintura de parasitos, } \\
\text { leitura de textos literários sobre } \\
\text { parasitos, jogo de trilha e jogo da } \\
\text { memória. }\end{array}$ \\
\hline $\begin{array}{c}2016 \\
\text { Maranhão }\end{array}$ & $\begin{array}{l}\text { Educação em } \\
\text { saúde como } \\
\text { instrumento de } \\
\text { prevenção das } \\
\text { parasitoses } \\
\text { intestinais no } \\
\text { município de } \\
\text { Grajaú - MA }\end{array}$ & $\begin{array}{c}\text { Gomes, S.C.S. } \\
\text { et al. }\end{array}$ & $\begin{array}{c}\text { Levar conhecimento a comunidades } \\
\text { periféricas a respeito das parasitoses } \\
\text { intestinais e seus efeitos na população } \\
\text { humana, enfatizando as ações de } \\
\text { higiene pessoal e saneamento básico } \\
\text { como fatores de prevenção para tais } \\
\text { doenças. }\end{array}$ & $\begin{array}{l}\text { Unidade } \\
\text { Básica } \\
\text { de } \\
\text { Saúde/ } \\
\text { Adultos } \\
\text { e } \\
\text { crianças }\end{array}$ & $\begin{array}{l}\text { Atividade Educativa em saúde } \\
\text { através de ações lúdica e rodas de } \\
\text { conversas após diagnóstico } \\
\text { situacional em crianças e adultos. }\end{array}$ \\
\hline $\begin{array}{c}2017 \\
\text { Minas Gerais }\end{array}$ & $\begin{array}{l}\text { Parasitoses } \\
\text { intestinais e } \\
\text { pediculose: } \\
\text { prevenção em } \\
\text { crianças na idade } \\
\text { escolar }\end{array}$ & $\begin{array}{l}\text { Novaes, } \\
\text { A.K.B. et al }\end{array}$ & $\begin{array}{l}\text { Relatar a experiência das atividades de } \\
\text { um projeto de extensão que tinha como } \\
\text { principal proposta à prevenção de } \\
\text { parasitoses intestinais e pediculose } \\
\text { desenvolvidas em escolares do } \\
\text { município de Juiz de Fora. }\end{array}$ & $\begin{array}{l}\text { Escola/ } \\
\text { Crianças }\end{array}$ & $\begin{array}{l}\text { Atividade Educativa lúdica, } \\
\text { através de jogos, vídeos e } \\
\text { palestras. }\end{array}$ \\
\hline
\end{tabular}




\begin{tabular}{|c|c|c|c|c|c|}
\hline $\begin{array}{c}2017 \\
\text { São Paulo }\end{array}$ & $\begin{array}{l}\text { Enteroparasitoses } \\
\text { em crianças de } \\
\text { uma creche na } \\
\text { cidade de Assis/SP } \\
\text { - Antes e Depois } \\
\text { de campanhas } \\
\text { educativas }\end{array}$ & $\begin{array}{l}\text { Ludwig, K.M.; } \\
\text { Conte, A.O.C. }\end{array}$ & $\begin{array}{l}\text { Realizar um levantamento da } \\
\text { prevalência de enteroparasitos em } \\
\text { alunos de uma creche da cidade de } \\
\text { Assis-SP, junto a campanhas } \\
\text { educativas sobre métodos de } \\
\text { profilaxia. }\end{array}$ & $\begin{array}{l}\text { Escola/ } \\
\text { Crianças }\end{array}$ & $\begin{array}{c}\text { Atividade Educativa através de } \\
\text { campanhas de educação sanitária } \\
\text { e profilática. }\end{array}$ \\
\hline $\begin{array}{c}2017 \\
\text { Paraíba }\end{array}$ & $\begin{array}{l}\text { Intervenção } \\
\text { educativa no } \\
\text { conhecimento das } \\
\text { geo-helmintíases } \\
\text { em escola } \\
\text { municipal }\end{array}$ & $\begin{array}{l}\text { Lima, } \\
\text { C.M.B.L. et al. }\end{array}$ & $\begin{array}{l}\text { Avaliar o impacto de estratégias } \\
\text { educativas, relacionadas a temas } \\
\text { pertinentes à parasitologia humana, no } \\
\text { conhecimento das principais geo- } \\
\text { helmintíases, ascaridíase e } \\
\text { ancilostomíase, em estudantes do } \\
\text { ensino fundamental, de uma escola } \\
\text { municipal. }\end{array}$ & $\begin{array}{l}\text { Escola/ } \\
\text { Crianças }\end{array}$ & $\begin{array}{l}\text { Atividade Educativa através de } \\
\text { apresentação, aula e brincadeiras. }\end{array}$ \\
\hline $\begin{array}{c}2018 \\
\text { Minas Gerais }\end{array}$ & $\begin{array}{l}\text { Promoção de saúde } \\
\text { na perspectiva da } \\
\text { prevenção de } \\
\text { doenças } \\
\text { parasitárias por } \\
\text { meio da educação } \\
\text { em saúde com } \\
\text { escolares do ensino } \\
\text { fundamental }\end{array}$ & $\begin{array}{l}\text { Dias, E.G. et } \\
\text { al. }\end{array}$ & $\begin{array}{l}\text { Relatar a experiência de uma ação de } \\
\text { educação em saúde com escolares do } \\
\text { ensino fundamental de uma escola } \\
\text { pública em zona rural. }\end{array}$ & $\begin{array}{l}\text { Escola/ } \\
\text { Adultos } \\
\text { e } \\
\text { Crianças }\end{array}$ & $\begin{array}{l}\text { Atividade Educativa através de } \\
\text { exposição dialógica do conteúdo } \\
\text { e exibição de vídeo disparador de } \\
\text { discussão; e roda de conversas. }\end{array}$ \\
\hline $\begin{array}{c}2018 \\
\text { Pernambuco }\end{array}$ & $\begin{array}{l}\text { Ações de } \\
\text { promoção da saúde } \\
\text { em feiras } \\
\text { municipais: um } \\
\text { relato de } \\
\text { experiência }\end{array}$ & $\begin{array}{l}\text { Martins, } \\
\text { V.H.S. et al. }\end{array}$ & $\begin{array}{l}\text { Relatar as experiências de estudantes } \\
\text { de enfermagem no desenvolvimento de } \\
\text { atividades de um projeto de extensão } \\
\text { intitulado "Conscientização sobre } \\
\text { doenças parasitárias em feiras } \\
\text { municipais de Petrolina/PE: A } \\
\text { universidade além dos muros". }\end{array}$ & $\begin{array}{l}\text { Feira/ } \\
\text { Adultos }\end{array}$ & $\begin{array}{l}\text { Atividade Educativa através de } \\
\text { ações lúdicas com jogos, teatros, } \\
\text { músicas, literatura em cordel, } \\
\text { vestimentas e/ou cartazes. }\end{array}$ \\
\hline Goiás & $\begin{array}{l}\text { Ações de educação } \\
\text { em saúde sobre } \\
\text { parasitoses } \\
\text { humanas em } \\
\text { escolas públicas no } \\
\text { município de Jataí, } \\
\text { Goiás }\end{array}$ & $\begin{array}{l}\text { Barcelos, } \\
\text { I.S.C. et al. }\end{array}$ & $\begin{array}{l}\text { Realizar ações educativas para } \\
\text { estudantes de escolas públicas sobre } \\
\text { parasitoses intestinais, pediculose, } \\
\text { toxoplasmose e tricomoníase com foco } \\
\text { na prevenção e controle de doenças } \\
\text { parasitárias. }\end{array}$ & $\begin{array}{l}\text { Escola/ } \\
\text { Crianças }\end{array}$ & $\begin{array}{l}\text { Atividade Educativa através de } \\
\text { palestras, cartazes, jogo de } \\
\text { memória, exibição de filmes e } \\
\text { teatro de fantoches. }\end{array}$ \\
\hline $\begin{array}{l}2019 \\
\text { Ceará }\end{array}$ & $\begin{array}{c}\text { Intervenção } \\
\text { educativa sobre } \\
\text { higienização das } \\
\text { mãos para crianças } \\
\text { na prevenção de } \\
\text { parasitoses }\end{array}$ & $\begin{array}{l}\text { Silva, A.K. et } \\
\text { al. }\end{array}$ & $\begin{array}{l}\text { Realizar intervenções de educação em } \\
\text { saúde com crianças do segundo ano do } \\
\text { ensino fundamental I sobre as } \\
\text { parasitoses intestinais. }\end{array}$ & $\begin{array}{l}\text { Escola/ } \\
\text { Crianças }\end{array}$ & $\begin{array}{l}\text { Atividade Educativa de através } \\
\text { de ações lúdicas de teatro de } \\
\text { fantoches, cartazes e figuras } \\
\text { ilustrativas, dinâmicas e vídeos. }\end{array}$ \\
\hline $\begin{array}{c}2019 \\
\text { Minas Gerais }\end{array}$ & $\begin{array}{l}\text { O estudo das } \\
\text { enteroparasitoses } \\
\text { como subsídio para } \\
\text { melhoria do ensino } \\
\text { de Biologia e } \\
\text { promoção da saúde }\end{array}$ & Silva, E.M.M. & $\begin{array}{l}\text { Utilizar o tema enteroparasitoses e sua } \\
\text { prevenção para estimular os alunos a } \\
\text { protagonizarem seu aprendizado sobre } \\
\text { tal conteúdo. }\end{array}$ & $\begin{array}{l}\text { Escola/ } \\
\text { Crianças }\end{array}$ & $\begin{array}{c}\text { Atividade Educativa através de } \\
\text { produção de ações pedagógicas } \\
\text { (aplicação de questionários, } \\
\text { produção de informativos, "Sala } \\
\text { Temática" e dia "D". }\end{array}$ \\
\hline $\begin{array}{c}2020 \\
\text { Paraíba }\end{array}$ & $\begin{array}{l}\text { Promoção de saúde } \\
\text { e combate das } \\
\text { parasitoses } \\
\text { intestinais através } \\
\text { de atividades } \\
\text { socioeducativas: }\end{array}$ & $\begin{array}{l}\text { Oliveira, I.B. } \\
\text { et al }\end{array}$ & $\begin{array}{c}\text { Educação para saúde com } \\
\text { contextualização socioeconômica e } \\
\text { cultural acerca das parasitoses } \\
\text { intestinais através de atividades como } \\
\text { palestras, oficinas e panfletagem. }\end{array}$ & $\begin{array}{l}\text { Escola e } \\
\text { UBS/ } \\
\text { Adultos } \\
\text { e } \\
\text { Crianças }\end{array}$ & $\begin{array}{l}\text { Atividade Educativa de sessões } \\
\text { de grupos de educação para a } \\
\text { saúde, através de oficinas lúdicas } \\
\text { para crianças, palestras para } \\
\text { adultos na UBS e visitas }\end{array}$ \\
\hline
\end{tabular}




\begin{tabular}{|c|c|c|c|c|c|}
\hline & $\begin{array}{l}\text { Relato de } \\
\text { experiência }\end{array}$ & & & & escolares. \\
\hline $\begin{array}{l}2020 \\
\text { Rio de } \\
\text { Janeiro }\end{array}$ & $\begin{array}{l}\text { Parasitoses } \\
\text { Intestinais: } \\
\text { Propostas de } \\
\text { Atividades } \\
\text { Lúdicas para o } \\
\text { Ensino } \\
\text { Fundamental II }\end{array}$ & $\begin{array}{c}\text { Faria, R.P. et } \\
\text { al }\end{array}$ & $\begin{array}{l}\text { Avaliar o impacto de estratégias } \\
\text { educacionais através do uso de } \\
\text { cartilhas e jogo de tabuleiro, levando } \\
\text { em consideração os conhecimentos e } \\
\text { crendices pré-estabelecidas no âmbito } \\
\text { escolar, }\end{array}$ & $\begin{array}{l}\text { Escola/ } \\
\text { Crianças }\end{array}$ & $\begin{array}{l}\text { Atividade Educativa através de } \\
\text { aplicação de jogo lúdico. }\end{array}$ \\
\hline $\begin{array}{l}2020 \\
\text { Pará }\end{array}$ & $\begin{array}{l}\text { Educação em } \\
\text { saúde para o } \\
\text { combate de } \\
\text { enteroparasitoses } \\
\text { em crianças de } \\
\text { uma creche } \\
\text { filantrópica em } \\
\text { Belém-PA: relato } \\
\text { de experiência }\end{array}$ & $\begin{array}{l}\text { Corrêa, } \\
\text { T.M.G. et al. }\end{array}$ & $\begin{array}{l}\text { Desenvolver atividades de educação } \\
\text { em saúde, visando o combate as } \\
\text { enteroparasitoses em crianças } \\
\text { atendidas em uma creche filantrópica } \\
\text { em Belém-PA }\end{array}$ & $\begin{array}{l}\text { Escola/ } \\
\text { Adultos } \\
\text { e } \\
\text { Crianças }\end{array}$ & $\begin{array}{c}\text { Atividade Educativa através de } \\
\text { roda de conversa sobre higiene } \\
\text { pessoal e ações lúdicas de teatro } \\
\text { de fantoches, pintura e confecção } \\
\text { de murais. }\end{array}$ \\
\hline $\begin{array}{c}2020 \\
\text { Minas Gerais }\end{array}$ & $\begin{array}{l}\text { Atividades de } \\
\text { prevenção para } \\
\text { pediculose e } \\
\text { parasitoses } \\
\text { intestinais em } \\
\text { escolares }\end{array}$ & $\begin{array}{l}\text { Lobo, N.N.M. } \\
\text { et al. }\end{array}$ & $\begin{array}{l}\text { Trabalhar estratégias lúdicas eficazes } \\
\text { de prevenção contra pediculose e } \\
\text { parasitoses intestinais dentro do } \\
\text { contexto escolar. }\end{array}$ & $\begin{array}{l}\text { Escola/ } \\
\text { Crianças }\end{array}$ & $\begin{array}{l}\text { Atividade Educativa através de } \\
\text { exibição de filmes, jogos e } \\
\text { construção de informativos. }\end{array}$ \\
\hline $\begin{array}{c}2020 \\
\text { Amazonas }\end{array}$ & $\begin{array}{l}\text { Educação em } \\
\text { saúde em uma } \\
\text { escola infantil do } \\
\text { interior do } \\
\text { Amazonas: Relato } \\
\text { de experiência }\end{array}$ & $\begin{array}{l}\text { Costa, A.M.S. } \\
\text { et al. }\end{array}$ & $\begin{array}{l}\text { Descrever a experiência vivenciada por } \\
\text { acadêmicas de enfermagem acerca da } \\
\text { realização de educação em saúde em } \\
\text { uma Escola de Ensino Infantil do } \\
\text { Interior do Amazonas. }\end{array}$ & $\begin{array}{l}\text { Escola/ } \\
\text { Crianças }\end{array}$ & $\begin{array}{l}\text { Atividade Educativa através de } \\
\text { teatro de fantoches, vídeos } \\
\text { didáticos e autoexplicativos. }\end{array}$ \\
\hline
\end{tabular}

Fonte: Autores.

Dos dezoito estudos realizados no Brasil selecionados para a revisão, 44,44\% dos artigos foram publicados na região Sudeste, representada pelos Estados de São Paulo (01), Rio de Janeiro (03), Minas Gerais (04); 33,33\% na região Nordeste, nos Estados do Maranhão (02), Pernambuco (01), Ceará (01) e Paraíba (02); 11,11\% na região Norte, nos Estados do Amazonas (01) e do Pará (01); 11,11\% na região Centro Oeste, nos Estados do Mato Grasso (01) e Goiás (01). Na região Sul não foram encontrados nenhum tipo de publicação referente à temática do estudo, apesar da literatura apontar que essa região não é isenta de condições socioeconômicas e ambientais relacionadas com a ocorrência de doenças endêmicas, incluindo as parasitoses intestinais (Moraes et al, 2019), mesmo considerando que a Região Sul do país apresenta temperaturas mais baixas do que as demais Regiões (Rey, 2008).

Todos os artigos foram publicados na língua portuguesa, sendo seus principais autores brasileiros. Em relação à distribuição temporal das publicações, a maioria dos trabalhos foi disponibilizado nos anos mais recentes, entre 2016 e 2020 , podendo demosntrar uma preocupação contínua dos pesquisadores em relação à utilização das estratégias de Promoção da Saúde em suas ações educativas sobre as enteroparasitoses no país. Foram publicados 27,78\% dos artigos no ano de 2020 , 16,67\% dos artigos nos anos de 2019, 2017, 2016 e 14,28\% 11,11\% dos artigos no ano de 2015.

Em todos os estudos encontrados observou-se a utilização de ações de educação em saúde como forma de Promoção da Saúde. Isso se justifica, porque, a educação em saúde é apontada como um instrumento profilático efetivo, de baixo custo e de fácil desenvolvimento por profissional de qualquer formação, seja ele da área da educação ou da saúde (Brasil, 2005; 
Toscani et al., 2007; Barbosa et al., 2009; Silva et al., 2014; Gomes et al., 2016).

A literatura tem mostrado uma tendência na utilização de práticas de educação em saúde como instrumento para a Promoção da Saúde em diferentes espaços profissionais de atuação (Toscani et al., 2007; Silva et al., 2014; Trindade et al., 2015). Outros espaços que ultrapassam os limites dos hospitais ou Unidades Básicas de Saúde também tem se destacado e cada vez mais tem recebido a atenção dos diferentes profissionais que lidam com problemas de Saúde Pública. Assim, as comunidades, as associações de moradores, as igrejas e as escolas são locais privilegiados, comumente escolhidos para o desenvolvimento de diferentes ações de Promoção da Saúde (Barbosa et al., 2009; Silva et al., 2014; Souza et al., 2015; Gomes et al., 2016). Essa tendência também foi evidenciada em nosso estudo.

Como exemplo dessa utilização de espaços alternativos, Oliveira et al (2020) destaca:

Ao considerar a escola como um dos pilares na formação de cidadãos pensantes e conscientes, ela funciona como um espaço propício para a educação em saúde, pois promove o conhecimento como principal mediador na busca por prevenção e tratamento às diversas enfermidades (Oliveira et al., 2020, p. 06).

As ações de promoção da saúde relatadas nas publicações que compuseram a presente revisão foram desenvolvidas, em sua grande maioria, no contexto escolar $(88,89 \%)$ e unidade básica de saúde $(11,11 \%)$, junto à população infanto-juvenil, em algum momento, acompanhada pelos seus pais e/ou responsáveis, professores e cuidadores (Araújo et al., 2015; Henrique et al., 2015; Teixeira 2016; Gomes et al., 2016; Siqueira 2016; Novaes et al., 2017; Ludwig; Conte, 2017; Lima et al., 2017; Dias et al., 2018; Barcelos et al., 2019; Silva et al., 2019; Silva, 2019; Faria et al., 2020, Corrêa et al., 2020; Lobo et al., 2020; Costa et al., 2020; Oliveira et al, 2020). As exceções foram os estudos de Martins et al (2018) desenvolvido no contexto comunitário (5,56\%), tendo como público os frequentadores e vendedores das feiras livres municipais, os quais puderam participar ativamente da produção de respostas criativas e eficazes para solucionar os problemas das parasitoses que foram abordados durante as atividades, e o de Dias et al (2018) que foi realizado em uma comunidade rural de descendentes de povos Quilombolas (5,56\%).

As estratégias de educação em saúde dirigidas à criança são preconizadas pelos Parâmetros Curriculares Nacionais PCN (Brasil, 1998), sendo as parasitoses intestinais temática curricular obrigatória inserida no currículo básico de ciências. A literatura aponta que por serem as crianças um público que apresenta alta prevalência de infecções parasitárias, esta é uma temática que merece ser discutida de forma interdisciplinar e multiprofissional, tanto no cotidiano escolar, já que este é um ambiente que também pode influenciar no processo saúde-doença, quanto no cotidiano da saúde coletiva (Toscani et al., 2007; Barbosa et al., 2009; Silva et al., 2014; Trindade et al., 2015; Gomes et al., 2016; Faria et al., 2020).

Sobre a importância da educação em saúde no contexto escolar, Faria et al (2020 destaca que:

O desenvolvimento cognitivo dos alunos mostra-se mais eficiente com as práticas educacionais aplicadas no seu cotidiano. Na ponte de integração entre essas duas vertentes, está o professor, que possui um papel extremamente importante, como mediador, no desenvolvimento do aluno como indivíduo (Faria et al., 2020, p. 236)

As ações educativas promovidas no ambiente escolar podem estimular intervenções que contribuam para a prevenção das parasitoses intestinais e para "uma aprendizagem efetiva e transformadora de atitudes e hábitos de vida" (Brasil, 1998, p. 245).

Nesse contexto, a educação em saúde deve colocada como "ferramenta importante para o compartilhamento de informações sobre a transmissão, sintomatologia, diagnóstico, tratamento, prevenção entre diferentes profissionais $e$ indivíduos vulneráveis as parasitoses intestinais" (Teixeira, 2016, p. 33).

Vale ressaltar que, o âmbito escolar deve ser corresponsável pela formação dos sujeitos para o entendimento e enfrentamento dos problemas, subsidiando-os na educação para a vida. Quando são investidos recursos em ações de promoção 
da saúde, concretamente em ações de educação em saúde, tende-se a produzir redução de sofrimento e de gastos financeiros nesse enfrentamento (Czeresnia \& Freitas, 2003; Brasil, 2010).

O direito ao acesso à informações corretas e que promovam a participação nos processos de tomadas de decisão em relação à condição de saúde, é um caminho que profissionais da educação e da saúde têm buscado em seus cotidianos para garantir que o tema das doenças parasitárias possa ser trabalhado com diferentes populações. Dessa forma, observou-se em todas as publicações encontradas a utilização de estratégias educativas que promovessem a ampliação do conhecimento parasitológico (Araújo et al., 2015; Henrique et al., 2015; Teixeira, 2016; Gomes et al., 2016; Siqueira, 2016; Novaes et al., 2017; Ludwig \& Conte, 2017; Lima et al., 2017; Dias et al., 2018; Martins et al., 2018; Barcelos et al., 2019; Silva et al., 2019; Silva, 2019; Faria et al., 2020, Corrêa et al, 2020; Lobo et al., 2020; Costa et al., 2020; Oliveira et al., 2020). Esses trabalhos mostram que há possibilidades para a ação educativa não se restringir ao mero repasse da informação mnemônica sobre a sintomatologia e tratamento, por exemplo.

Importante destacar os trabalhos que relatam a abordagem de informações referentes ao ciclo de vida e formas de contágio dos parasitos (Araújo et al., 2015; Siqueira, 2016; Novaes et al., 2017; Martins et al., 2018; Barcelos et al., 2019; Silva 2019; Corrêa et al., 2020), representando a valorização de estratégias que permitem maior dinamismo e interação dos indivíduos com os conteúdos parasitológicos, possibilitando aprendizados significativos sobre as formas infectantes e contextualizados com a realidade de vida e consequente exposição ao risco de aquisição das mesmas no ambiente precário.

O uso de estratégias lúdicas, como jogos, teatros de fantoches, músicas, literatura, brincadeiras e metodologias dinâmicas com uso de materiais didáticos com figuras de parasitos, vídeos, montagens de macromodelos ou oficinas de demonstrações de espécimes conservados em álcool $70 \%$ ou formol, se traduziu em metodologias inovadoras utilizadas principalmente no ambiente escolar (Araújo et al., 2015; Henrique et al., 2015; Teixeira, 2016; Gomes et al., 2016; Siqueira, 2016; Novaes et al., 2017; Lima et al., 2017; Dias et al., 2018; Martins et al., 2018; Barcelos et al., 2019; Silva et al., 2019; Silva, 2019; Faria et al., 2020; Corrêa et al., 2020; Lobo et al., 2020; Costa et al., 2020; Oliveira et al., 2020), sendo possível despertar o interesse de crianças e adultos para o ato preventivo e para disseminação das informações no meio social em que vivem. De acordo com os estudos, as crianças demonstram essa relação ao elaborarem conceitos sobre prevenção das parasitoses não somente na sala de aula, mas, também em seus domicílios.

A importância da utilização de estratégias diferenciadas, inovadoras e simples é destacada por Henrique et al (2015):

Compreendemos que o uso de metodologias diferenciadas para o ensino de qualquer área, não é tarefa fácil e muitas vezes não é encarada de maneira positiva, porém observamos na prática que o ensino de temas voltados à realidade e cotidiano do aluno, como conteúdos referentes à saúde, mais precisamente, verminoses, torna a aprendizagem mais significativa, mesmo com uma modalidade didática simples (Henrique et al., 2015, p. 03).

Ainda sobre o uso de estratégias lúdicas, Toscani et al (2007) e Trindade et al (2015) relatam que as mesmas, além de serem bem aceitas pelos estudantes, são acessíveis, devido ao baixo custo e se mostram tão eficazes quanto ações diretivas como o saneamento básico, podendo produzir aprendizados significativos. O lúdico, segundo Faria et al (2020), é ferramenta importante para a interação entre o intelecto e personalidade e o pleno desenvolvimento do organismo de uma criança. Os jogos, por exemplo, podem auxiliar no desenvolvimento cognitivo e na aprendizagem significativa das doenças parasitológicas, estimulando o raciocínio através da criação de relações entre o conteúdo transmitido e situações do cotidiano.

Os jogos e as atividades lúdicas "podem auxiliar os professores a somar positivamente na familiarização do conteúdo transmitido através da criação de um momento agradável e instigante, tornando o aprendizado mais fácil e eficaz" (Lobo et al., 2020, p. 06). Entretanto, Toscani et al (2007) e Trindade et al (2015) chamam a atenção para o cuidado em relação ao equilíbrio necessário entre a função lúdica e pedagógica para que seja conciliado a liberdade do jogar e a orientação do processo educativo desejado. 
Além dessas estratégias, Silva (2019) e Lobo et al (2020) propõem a produção, pelos alunos, de materiais informativos (cartazes e banners) sobre as parasitoses intestinais para serem utilizados também no ambiente escolar, como por exemplo, nas feiras escolares. A construção de campanhas educativas sobre as parasitoses foi a estratégia realizada por Ludwig \& Conte (2017) para promover a divulgação de informações relacionadas à profilaxia.

Esses recursos didáticos são apontados pela literatura levantada como facilitadores para o ensino-aprendizagem das parasitoses junto ao público infantil, visto que estimulam o interesse, melhoram a iniciativa, a forma de comunicação e a criatividade (Toscani et al., 2007; Trindade et al, 2015). Além disso, esses recursos são de boa receptividade por parte dos escolares, pois, ao abordarem temas relacionados aos hábitos saudáveis de higienização pessoal e limpeza dos alimentos antes da ingestão, reapresentam acontecimentos próximos da realidade das crianças, fazendo mais sentido ao seu aprendizado (Araújo et al., 2015; Henrique et al., 2015; Teixeira, 2016; Gomes et al., 2016; Siqueira, 2016; Novaes et al., 2017; Lima et al., 2017; Dias et al., 2018; Martins et al., 2018; Barcelos et al., 2019; Silva et al., 2019; Faria et al., 2020, Corrêa et al., 2020; Lobo et al., 2020; Costa et al., 2020; Oliveira et al., 2020). No mesmo raciocínio, Araújo et al (2015) complementa afirmando que o conhecimento só se tornará efetivo quando for significativo para os alunos e quando for percebido como um assunto de interesse comum.

Acredita-se que as crianças possam ser capazes de transmitir conhecimento e ao longo dos tempos, transformarem sua realidade a partir do acesso à informação correta em saúde [...]. Considerou-se eficaz a realização da educação em saúde, visto que a população é carente de conhecimento e necessita interação e oportunidade de se expressarem sobre suas necessidades de saúde (Dias et al., 2018, p. 03).

As metodologias roda de conversa, grupo focal e ou palestras também foram estratégias utilizadas por Teixeira (2016), Gomes et al (2016), Dias et al (2018), Barcelos et al (2019), Corrêa et al (2020) e Oliveira (2020) para trabalhar o tema das infecções enteroparasitárias e os aspectos relativos aos hábitos de higiene saudáveis preventivos como a lavagem das mãos, uso de calçados, lavagem e preparo dos alimentos.

Através da utilização do grupo focal, no terceiro e último momento das atividades educacionais, as crianças mostraram que aprenderam sobre o conhecimento compartilhado através das atividades de educação em saúde. As crianças conseguiram verbalizar sobre as parasitoses intestinais, formas de transmissão, mecanismos de prevenção, local da infecção no hospedeiro, bem como conceitos específicos como o nome dos parasitos: lombriga, $A$. lumbricoides, e Giárdia (Teixeira, 2016, p. 42).

Essas metodologias mostram-se eficazes, pois, permitem a discussão da saúde como uma questão coletiva, favorecendo a troca de experiências através do debate sobre as diferentes respostas cotidianas individuais e coletivas relacionadas aos problemas e as estratégias preventivas para as parasitoses no contexto das famílias. No entanto, Siqueira (2016) chama a atenção para a importância da continuidade multiplicadora das atividades nas escolas, visto que o desenvolvimento de ações pontuais podem não propiciar a fixação das informações, tanto pelos professores, quanto pelos estudantes.

Castellanos (1997) discute que as necessidades e os problemas que permeiam a vida tem origem nas interações e interrelações vividas pelos indivíduos. Para o autor, essas necessidades e problemas demandam respostas, que no caso específico da situação de saúde, têm como objetivo modificar, evitar ou minimizar os impactos sobre a saúde individual e coletiva. Nessa perspectiva, é importante entender que toda ação educativa em saúde deve considerar as diferentes respostas construídas para solucionar os problemas cotidianos. Caso contrário essas ações se manterão contrárias ao sentido educacional, sendo consideradas autoritárias, verticais, desestimulantes, não produzindo significados necessários a mudanças efetivas pelos indivíduos. 
No trabalho profilático das parasitoses é essencial que as ações educativas em saúde também sejam aliadas ao diagnóstico precoce e ao tratamento medicamentoso acessível e eficaz. Dessa forma, observou-se que algumas das ações relatadas nos trabalhos ocorreram somente após um diagnóstico situacional da ocorrência das infecções parasitárias, sento este discutido junto com a população (Siqueira, 2016; Gomes et al., 2016; Ludwig \& Conte, 2017), de modo mais participativo, pois, a comunidade deve estar integrada, sendo informada sobre a problemática e tendo participação ativa nas soluções (REY, 2008).

Com isso, a visão de promoção de saúde possibilita a construção de processos de intervenção de forma compartilhada e corresponsabilizada, distanciados de modelos tradicionais e da simples transmissão de informação (Sícoli \& Nascimento, 2003).

A promoção da saúde envolve o fortalecimento da capacidade de escolha consciente, requerendo um conhecimento ampliado sobre as questões de diferenças e singularidades dos acontecimentos que envolvem o processo saúde doença. Ademais, requer o reconhecimento de que o ambiente de vida é consolidado através de trajetos próprios e únicos, sendo expressos por meio das situações concretas que não podem ser desconsideradas (Castellanos, 1997; Czeresnia \& Freitas, 2003; Bus, 2009).

Nessa lógica, a saúde deve ser compreendida como produto social, sendo condicionada por diferentes acontecimentos e necessitada, para que existam melhorias na qualidade de vida, da participação ativa de todos. Sendo a educação em saúde uma das ferramentas indispensáveis ao trabalho preventivo e de controle das parasitoses intestinais, esta não poderá ter seu enfoque menosprezado e/ou limitado ao nível individual, se atendo também na responsabilidade que o coletivo exerce.

Como limitação deste estudo, destacamos a dificuldade do encontro de pesquisas que relatam experiências práticas de ações educativas direcinadas às parasitoses intestinais. Sendo assim, é importante que novas pesquisas sejam criadas de modo a explorar melhor o assunto.

As contribuições desta revisão apoiam-se na possibilidade da divulgação de trabalhos importantes, caracterizados como literatura cinzenta (teses e dissertações), bem como divulgar a importância da Educação em Saúde, que fortaleçam futuras pesquisas envolvendo a temática.

\section{Considerações Finais}

Os estudos apresentados nesta revisão concordam com o fato de que as parasitoses intestinais ainda sejam doenças negligenciadas, carentes de discussão no campo da Promoção da Saúde, especificamente como tema que deve ser abordado por meio de ações de Educação em Saúde. Com isso, este estudo conclui que poucos, ainda, são os trabalhos que, efetivamente, se dedicam a inserir estratégias de Educação em Saúde, direcionadas para a prevenção e controle de enteroparasitoses em suas práticas. Isso tende a contradizer, exatamente, o fato das parasitoses intestinais serem importante problema de Saúde Pública, geralmente, associadas à precariedade das condições de vida da população.

De um modo geral, os autores trazem que a prevenção e o controle dessas infecções apoiam-se no investimento em saneamento básico, diagnóstico, tratamento direcionado e implementação de ações de Promoção da Saúde. Esta estratégia pode ser um importante caminho para renovação e reconfiguração de práticas em saúde que valorizem saberes para a promoção de qualidade de vida coletiva.

É importante destacar as limitações da atuação dos serviços de saúde no controle das parasitoses. Isolados, a tendência é que as ações não sejam realizadas ou não sejam eficazes. Isso porque, os problemas só poderão ser resolvidos a partir da integração corresponsável com áreas como saneamento, habitação, educação, dentre outras, necessárias para a melhoria das condições de vida da população. Considerando a saúde como um produto social de diferentes condicionantes, a participação 
ativa de todos os envolvidos em sua produção se torna indispensável para a construção de respostas eficientes e de melhorias contínuas.

É notório que as práticas educativas em saúde são estratégias indispensáveis para a promoção da saúde. Elas consistem em vias de mão dupla para ensinar e aprender, numa constante troca onde o objetivo central é transformar situações adversas e de vulnerabilidade em realidades onde haja condições de vida saudáveis e justas. Dessa forma, é importante o investimento em ações educativas em saúde nas escolas e em outros espaços coletivos que fortaleçam as capacidades autônoma, autorreflexiva e crítica dos indivíduos, favorecendo a tomada de decisões relativas à criação de respostas relacionadas não somente às parasitoses intestinais, mas, as outras necessidades e questões de saúde da comunidade.

\section{Referências}

Andrade, E. C., Leite, I. C. G., Rodrigues, V. O., \& Cesca, M. G. (2010). Parasitoses intestinais: uma revisão sobre seus aspectos sociais, epidemiológicos, clínicos e terapêuticos. Rev. APS, 13(2), 231-240. https://periodicos.ufjf.br/index.php/aps/article/view/14508/7809.

Araújo, A. A., Araújo, C. L., Sarmento, E. C., Paula, L. R., \& Santos, D. S. V. (2015). Prevenção a parasitoses ocasionadas por cestodas através de jogos lúdicos no ensino fundamental II. In: II Congresso nacional de Educação - II CONEDU: Políticas, Teorias e Prática. https://editorarealize.com.br/editora/anais/conedu/2015/TRABALHO_EV045_MD1_SA18_ID5909_08092015155159.pdf.

Barbosa, L. A., Sampaio, A. L. A., Melo, A. L. A., Macedo, A. P. N., \& Machado, M. F. A. S. (2009). A educação em saúde como instrumento na prevenção de parasitoses. RBPS, 22(4), 272-278. https://doi.org/10.5020/1048.

Barcelos, I. S. C., Almeida, L. F., Oliveira, A. A., \& Rodrigues, R. M. (2019). Ações de educação em saúde sobre parasitoses humanas em escolas públicas no município de Jataí, Goiás. Em Extensão, Uberlândia, 18(2). https://doi.org/10.14393/REE-v18n22019-49658.

Basso, R. M. C., Silva-Ribeiro, R. T., Soligo, D. S., Ribacki, S. I., Callegari-Jacques, S. M. 2 \& Zoppas, B. C. A. (2008). Evolução da prevalência de parasitoses intestinais em escolares em Caxias do Sul, RS. Revista Sociedade Brasileira Medicina Tropical, 41(3), 263-268. https://doi.org/10.1590/S003786822008000300008 .

Brasil (1998). Ministério da Educação. Parâmetros curriculares nacionais: Ciências Naturais/ Secretaria de Educação Fundamental. Brasília: Secretaria de Educação Fundamental, 1998. http://portal.mec.gov.br/seb/arquivos/pdf/ciencias.pdf.

Brasil (2002). Ministério da Saúde. As cartas da promoção da saúde. Brasília, Ministério da Saúde, 2002.https://bvsms.saude.gov.br/bvs/ publicacoes/cartas_promocao.pdf.

Brasil (2005). Ministério da Saúde. Plano Nacional de Vigilância e Controle das Enteroparasitoses. Secretaria de Vigilância em Saúde. Ministério da Saúde, 2005. https://bvsms.saude.gov.br/bvs/publicacoes/enteroparasitoses_pano_nacional.pdf.

Brasil (2010). Ministério da Saúde. Política Nacional de Promoção da Saúde. Secretaria de Vigilância em Saúde. Secretaria de Atenção à Saúde. (3a ed.), Brasília: Ministério da Saúde, 2010. https://bvsms.saude.gov.br/bvs/publicacoes/politica_nacional_promocao_saude_3ed.pdf.

Busato, M. A., Antoniolli, M. A., Teo, C. R. P. A., Ferraz, L., Poli, G., \& Tonini, P. (2014). Relação de parasitoses intestinais com as condições de saneamento básico. Ciência, Cuidado e Saúde, 13(2). https://doi.org/10.4025/cienccuidsaude.v13i2.18371.

Buss, P. M. (2003). Uma introdução ao conceito de promoção da saúde. In: Czeresnia, D. \& Freitas, C.M. (Org.). Promoção da saúde. Fiocruz.

Castellanos, P. L. (1997). Epidemiologia, saúde pública, situação de saúde e condições de vida: considerações conceituais, 31-76. In: Barata, R.B. (org.). Condições de Vida e Situação de Saúde. Saúde Movimento, 4. Abrasco, 1997.

Corrêa, T. M. G., Araujo, T. R., \& Arruda, J. E. G. (2020). Educação em saúde para o combate de enteroparasitoses em crianças de uma creche filantrópica em Belém - PA: relato de experiência. REAS / EJCH, 12(9). https://doi.org/10.25248/reas.e3883.2020.

Costa, A. M. S., Reis, D. A., Rocha, T. D. P., Gomes, Y. S., \& Mata, L. M. (2020) Educação em saúde em uma escola infantil do interior do Amazonas: Relato de experiência. REVISA, 9(1): 125-32. https://doi.org/10.36239/revisa.v9.n1.p125a132.

Czeresnia, D.; \& Freitas, C. M. Promoção da saúde: conceitos, reflexões, tendências. Editora FIOCRUZ, 2003.

Dias, E. G., Oliveira, C. K. N., Teixeira, J. A. L., Anjos, A. K. T., \& Lima, J. A. D. (208). Promoção de Saúde na perspectiva da rrevenção de doenças parasitárias entre escolares do ensino fundamental. Revista de Epidemiologia e Controle de Infecção, 8(3). http://dx.doi.org/10.17058/reci.v8i3.9958.

Faria, R. P., Carneiro, L. A. D., \& Neto, A. H. A. M. (2020). Parasitoses Intestinais: propostas de atividades Lúdicas para o ensino fundamental II. Ensino, Saúde e Ambiente, 13(3). https://periodicos.uff.br/ensinosaudeambiente/article/view/28670/28380.

Galvao, T. F., \& Pereira, M. G. (2014). Revisões sistemáticas da literatura: passos para sua elaboração. Epidemiol. Serv. Saúde, 23(1), 183-184. http://dx.doi.org/10.5123/S1679-49742014000100018.

Gomes, S. C. S., Rodrigues, S. R., Silva, A. B., Arruda, A. K. S., Silva, N. M., Macedo, R. S., Lima, E. N. P. \& Ferreira, I. E. A. (2016). Educação em saúde como instrumento de prevenção das parasitoses intestinais no município de Grajaú - MA. Pesquisa em Foco, 21(1). https://doi.org/10.18817/pef.v21i1.1123. 
Henrique, V. H. O. Alexandre, M., Almeida, Q. R., \& Sousa, P. S. (2015). Oficina educativa sobre verminoses: contribuição para Educação profilática na escola. $8^{\circ}$ Congresso de Extensão Universitária da UNESP: Diálogos da Extensão: do saber acadêmico à prática social. https://repositorio.unesp.br/handle/11449/142690.

Lima, C. M. B. L., Menezes, K. S., Ferreira, S. O., Santana, B. H., Fernandes, A. S. S., Rangel, V. S., Soares, D. A., \& Freitas, F. I. S (2017). Intervenção educativa no conhecimento das geo-helmintíases em escola municipal. Rev. Ciênc. Ext. 13(1). https://ojs.unesp.br/index.php/revista _proex/article/view/1375/0.

Lobo, N. N. M., Alves, A. C. A.,Amaral, D. L. A. S., \& Abramo, C. (2020). Atividades de prevenção para pediculose e parasitoses intestinais em escolares. Lynx, 1(1). https://doi.org/10.34019/2675-4126.2020.v1.31239.

Ludwig, K. M., Frei, F., Álvares Filho, F., \& Ribeiro-Paes, J. T. (1999). Correlação entre condições de saneamento básico e parasitoses intestinais na população de Assis, Estado de São Paulo. Rev. Soc. $\quad$ Bras. Med. $\quad$ Trop. 32 (5):547-555. https://www.scielo.br/j/rsbmt/a/HJpjBRS7VSVVypbwjHzkd8q/abstract/?lang=pt.

Ludwig, K. M., \& Conte, A. O. C. (2017). Enteroparasitoses em crianças de uma creche na cidade de Assis/SP - antes e depois de campanhas educativas. Revista Saúde (Santa Maria), 43(2). https://periodicos.ufsm.br/revistasaude/article/view/23158.

Mackey, T. K. Liang, B. A., Cuomo, R., Hafen, R., Brouwer, K. C., \& Lee, D. E. (2014). Emerging and reemerging neglected tropical diseases: a review of key characteristics, risk factors, and the policy and innovation environment. Clinical Microbiology Reviews, 27(4). https://pubmed.ncbi.nlm.nih.gov/25278579/.

Munareto, S., Lima, A. P. S., Zardeto-Sabec, G., Vieira, S. L. V. (2021). Parasitosis in preschool children in Brazil: bibliographic review. Research, Society and Development, 10, e1910111195. https://doi.org/10.33448/rsd-v10i1.11195.

Martins, V. H. S., Lima, K. M., Belfort, L. R. M., \& Bezerra, N. C. (2018). Ações de promoção da saúde em feiras municipais: um relato de experiência. Revista de Extensão da UNIVASF, 6(2). https://www.periodicos.univasf.edu.br/ind.

Melo, M. C. B., Klem, V. G. Q., Mota, J. A. C., \& Penna, F. J. (2004). Parasitoses Intestinais. Rev Med Minas Gerais; 14 (1): S3-S12. https://pesquisa.bvsalud.org/portal/resource/pt/lil-776029.

Minayo, M. C. S. (2007). O Desafio do Conhecimento: Pesquisa Qualitativa em Saúde. (10a ed.), HUCITEC.

Moraes, L. J. R. Andrade, L. S., Farias, C. B. P., \& Pinto, L. C. (2019). Prevalência de anemia associada a parasitoses intestinais no território brasileiro: uma revisão sistemática. Rev Pan-Amaz Saude, Ananindeua, 10, e201900098. http://dx.doi.org/10.5123/s2176-6223201900098 .

Neves, D. P. (2016). Parasitologia humana. (13a ed.), Editora Atheneu.

Novaes, A. K. B., Paiva,G. M. S., Ubaldino,A. C. C. Santos, L. M., \& Rosa, F. M. (2017). Parasitoses intestinais e pediculose: prevenção em crianças na idade escolar. Rev. APS, 20(3). https://doi.org/10.34019/1809-8363.2017.v20.16010.

Oliveira, I. B., Guimarães, J. F., Melo, M. P., Ferreira, J. F., Freires, L. S., Queiroz, L. I. S., \& Maranhense, P. S. A. Promoção de saúde e combate das parasitoses intestinais através de atividades socioeducativas: Relato de experiência. Braz. J. Hea. Rev., 3(4). 10.34119/bjhrv3n4-336.

Rey, L. (2008). Parasitologia. (4a ed.), Guanabara Koogan.

Sícoli, J. L., \& Nascimento, P. R. (2003). Promoção de saúde: concepções, princípios e operacionalização. Interface - Comunic, Saúde, Educ, 7(12), 91-112.

Silva, M. M. A., Lima, R. M., \& Bastos, S. N. D. (2014) Parasitoses intestinais do município de Nova Esperança do Piriá: conhecer para evitar. Ensino, Saúde e Ambiente, 7(1). https://doi.org/10.22409/resa2014.v7i1.a21185.

Silva, M. R., Lamas, L. R. R., Lana, P. P., Pinto, E. S. O. (2018). Educação em saúde sobre parasitoses: um relato de experiência. Revista Científica Univiçosa 10(1). https://academico.univicosa.com.br/revista/index.php/RevistaSimpac/article/view/1186.

Silva, A. K., Vasconcelos, B. M., Carvalho, K. G., Nery, M. T. R., Ferreira, N. G., Santos, N. S. P., Sá, R. K. A., Andrade, M. S. N., Castro, A. P. R., \& Medeiros, K. M. F. (2019). Intervenção educativa sobre higienização das mãos para crianças na prevenção de parasitoses. Revista Interfaces, 7(1).

Siqueira, M. P. (2016). Parasitoses intestinais em escolares de Niterói, RJ: frequência, conhecimentos e educação em saúde. Dissertação (Mestrado em Microbiologia e Parasitologia) - Universidade Federal Fluminense, Instituto Biomédico, Niterói, 124 f. https://sucupira.capes.gov.br/ sucupira/public/consultas/coleta/trabalhoConclusao/viewTrabalhoConclusao.jsf?popup=true\&id_trabalho=3898120.

Sousa, A. C. M., Bocardi, M. I. B., \& Cardoso, T. L. (2015). Hábitos de vida como fator desencadeante a parasitoses intestinais. Ideias \& Inovação.. 2. 77-92. https://periodicos.set.edu.br/ideiaseinovacao/article/view/2210.

Teixeira, P. A., Fantinatti, M., Gonçalves, M. P., \& Silva, J. S. (2020). Parasitoses intestinais e saneamento básico no Brasil: estudo de revisão integrativa. Braz. J. of Develop., 6, 22867-22890. 10.34117/bjdv6n5-006.

Teixeira, P. A. (2016). Conhecimentos sobre parasitoses intestinais como estratégia para subsidiar ferramentas de educação em saúde. Dissertação (Mestrado em Medicina Tropical) - Fundação Oswaldo Cruz, 106 f. https://www.arca.fiocruz.br/handle/icict/23212.

Toscani, N. V., Santos, A. J. D. S., Silva, L. L. M., Tonial, C. T., Chazan, M. Wiebbelling, A. M. P., \& Mezzari, A. (2007). Development and analysis of an educational game for children aiming prevention of parasitological diseases. Interface - Comunic., Saúde, Educ., 11(22), 281-94. https://doi.org/10.1590/S1414-32832007000200008

Trindade, F. F., Dantas, M. A. T., Donato, C. R., \& Vieira, F. S. (2015). Descobrindo as parasitoses: jogo educativo para o ensino de ciências. Educationis, Aquidabã, 2(1). 10.6008/ESS2318-3047.2014.001.0003. 